\title{
Biatrial Recurrence of Two Independently Growing Cardiac Myxoma in a Patient with Multiple Tumor Disease
}

\author{
Stefan R.B. Schneider ${ }^{1} \quad$ Angelo Dell'Aquila ${ }^{1} \quad$ Sven Martens $^{1} \quad$ Andreas Rukosujew $^{1}$ \\ ${ }^{1}$ Division of Cardiac Surgery, Department of Cardiothoracic Surgery, \\ Universitätsklinikum Münster, Münster, Germany \\ Thorac Cardiovasc Surg Rep 2014;3:35-37.

\begin{abstract}
Address for correspondence Stefan R.B. Schneider, MD, Division of Cardiac Surgery, Department of Cardiothoracic Surgery, Universitätsklinikum Münster, Albert-Schweitzer-Campus 1 Gebäude A1,
\end{abstract} \\ 48149 Münster, Germany (e-mail: stefan.schneider@ukmuenster.de).
}
Abstract
Keywords
- cardiac tumor
- cardiac myxoma

We report the case of a 56-year-old female patient with biatrial recurrence of cardiac myxoma and extensive comorbidities. In the literature, only few cases of biatrial myxoma can be found and they generally describe a single tumor reaching both atria. We found two independently growing cardiac myxomas of both atria.

\section{Introduction}

Tumors of the heart are rare with a frequency of 0.017 and $0.28 \%$ in autopsy series. Myxoma is the most common cardiac tumor, accounting for more than $70 \%$ of all cardiac neoplasms. ${ }^{1}$ Only a few cases of biatrial myxoma or biatrial recurrence of cardiac myxoma have been reported and they generally describe a single tumor reaching both atria. ${ }^{2}$ In this study, we found two independently growing atrial myxomas.

We report on a 56-year-old female patient who was referred to our center with diagnosis of a biatrial tumor mass in 2012. She had an extensive hospital record with Leiden factor $\mathrm{V}$ mutation, Leriche syndrome, tumor of unknown dignity of the uterus and the breasts, and history of removal of left atrial cardiac myxoma in 2008. Embolic Leriche syndrome had manifested as a consequence of a left atrial myxoma which was removed in a second step. During the first surgery in 2008 , both atria were inspected, and a left atrial tumor mass was found, originating from the left upper pulmonary vein outlet. It had been excised with a $1-\mathrm{cm}$ resection margin and the defect reconstructed using a Gore Tex (W.L. Gore \& Associates, Inc., Newark, Delaware, United States) patch. Histology had confirmed cardiac myxoma with a tumor-free resection margin. There was neither a family history of cardiac myxoma nor signs of a carney complex.

We performed a complete tumor staging including transesophageal echocardiography ( $\mathbf{F i g} . \mathbf{1}$ ) and computed to- mography (-Fig. 2). The left atrial tumor prolapsed into the left ventricle; there was no infiltration of surrounding structures, so a metastasis of the gynecologic tumors seemed unlikely. Finally, we decided to urgently remove the biatrial tumor mass to prevent further complications such as pulmonary embolism or stroke.

Because it was a redo surgery, the extracorporeal circulation was connected to the right axillary artery and the femoral vein, before we gained surgical access via median sternotomy. An additional cannula for venous drainage was connected to the superior caval vein, thus achieving complete bicaval bypass. The heart was arrested with cold blood cardioplegia. Opening the right atrium, we found it was completely filled with a 6-cm dark blue, polypous pedunculated tumor mass. The left atrium was opened through the interatrial septum. Inside we found a $7-\mathrm{cm}$, round, pedunculated tumor mass (-Fig. 3). Tumors were not connected and unlike the finding during the first surgery in 2008, the tumors originated from different parts of the interatrial septum and were not in touch with the old resection area. Both tumors were completely resected, including the adjacent parts of the interatrial septum (-Fig. 4). Atria were reconstructed using bovine pericardium.

The postoperative course was uneventful and the patient was referred to our gynecology unit for further treatment. Histology revealed cardiac myxoma without signs of malignancy. Resection margins were tumor free. For the further treatment course, we recommended echocardiographic received

November 17, 2013

accepted

December 27, 2013

published online

March 26, 2014
DOI http://dx.doi.org/

10.1055/s-0034-1371393. ISSN 2194-7635. (c) 2014 Georg Thieme Verlag KG
Stuttgart · New York

License terms

(®) $\Theta \circledast$ 


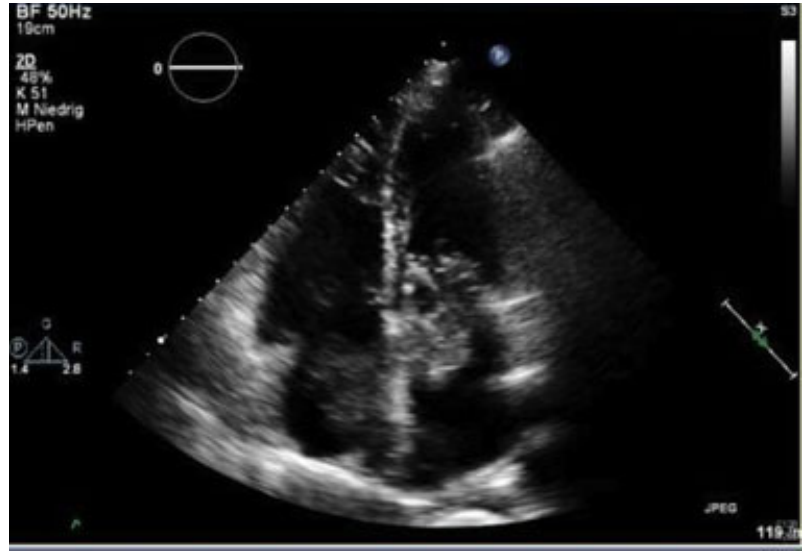

Fig. 1 Transthoracic echocardiography: four-chamber view showing the biatrial tumor mass.

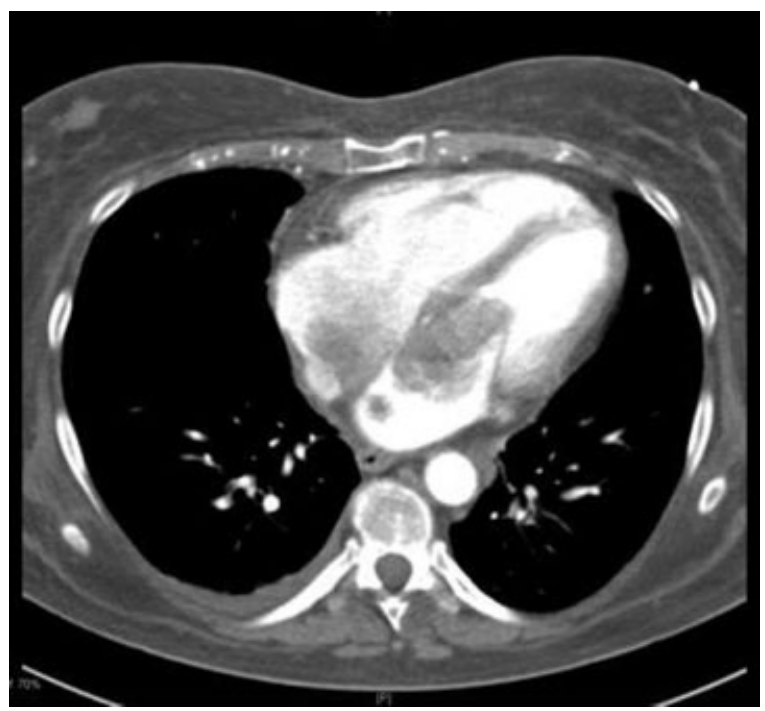

Fig. 2 Computed tomography: four-chamber view showing the biatrial tumor mass.

checkups on a regular basis to timely diagnose another recurrence of cardiac myxoma.

\section{Discussion}

The risk for recurrence of cardiac myxoma is thought to be approximately 1 to $3 \%$ for sporadic tumors and 12 to $22 \%$ for familial and complex myxomas; mostly recurrent myxomas appear within the first 4 years after primary surgery. ${ }^{3}$

In our case, the cause for the recurrence of the myxoma remains unclear. There were no typical signs of a carney complex-like skin lesions or hyperpigmentation. Different etiologies of myxoma recurrence including malignant metastasis are discussed in the literature. ${ }^{3-5}$ If the reason for the recurrence was an inadequate surgical resection at first surgery, then the question remains why tumor recurrence happened in both atria. A possible explanation might be

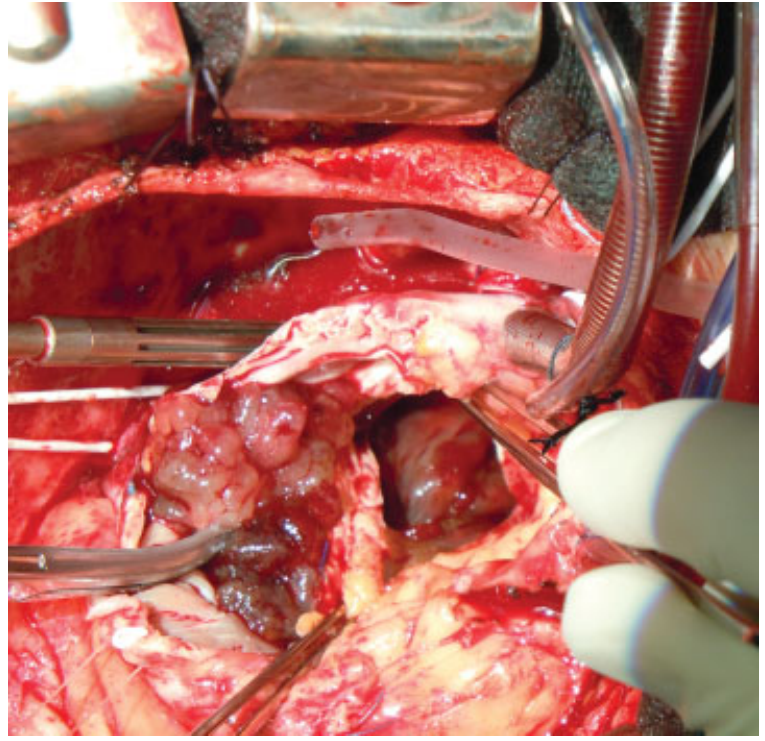

Fig. 3 Intraoperative picture of both myxomas in situ.

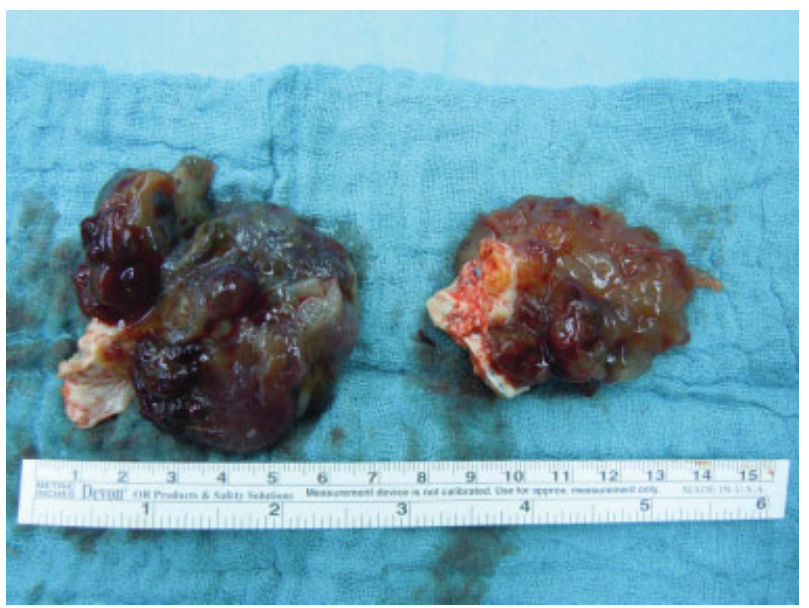

Fig. 4 Both tumors after resection.

spreading of tumor cells during the first surgery or a multilocalized tumor that has started growing after the first surgery and had not been detected. As the etiology of myxoma recurrence is unclear, all patients after surgical removal of cardiac myxoma should receive routine echocardiographic checkups.

Even in patients with complex multimorbidity, removal of cardiac tumors is possible with remarkable postoperative outcome. In our case, surgical removal of the tumor was necessary both in curative and palliative intent. Only with the cardiac problem solved, curative treatment of the gynecologic tumor became possible.

\section{Acknowledgments}

Authors acknowledge support by Deutsche Forschungsgemeinschaft and Open Access Publication Fund of University of Muenster. 


\section{Conflict of Interest}

None declared.

\section{References}

1 Hoffmeier A, Schmid C, Deiters S, et al. Neoplastic heart diseasethe Muenster experience with 108 patients. Thorac Cardiovasc Surg 2005;53(1):1-8
2 Voluckiene E, Norkūnas G, Kalinauskas G, et al. Biatrial myxoma: an exceptional case in cardiac surgery. J Thorac Cardiovasc Surg 2007;134(2):526-527

3 Reynen K. Cardiac myxomas. N Engl J Med 1995;333(24): $1610-1617$

4 Imperio J, Summers D, Krasnow N, Piccone VA Jr. The distribution patterns of biatrial myxomas. Ann Thorac Surg 1980;29(5):469-473

5 Seo IS, Warner TF, Colyer RA, Winkler RF. Metastasizing atrial myxoma. Am J Surg Pathol 1980;4(4):391-399 